\title{
Ultrasonographic features of aggressive primary thyroid diffuse B-cell lymphoma: A report of two cases
}

\author{
GUOBING HU and XIANGMING ZHU \\ Department of Ultrasound, The First Affiliated Hospital of Wannan Medical College, Wuhu, Anhui 241001, P.R. China
}

Received January 17, 2015; Accepted January 21, 2016

DOI: $10.3892 / 01.2016 .4255$

\begin{abstract}
Primary thyroid lymphoma (PTL) is a relatively rare malignant tumor. Aggressive PTL is extremely rare, and there is limited literature regarding the imaging features of PTL with invasion into adjacent structures, including internal jugular vein, muscles, esophagus, trachea and carotid artery. In addition, the ultrasonographic features of the cases presented in the current report differ from those reported in previous studies. In the present study, two cases of PTL, who presented to The First Affiliated Hospital of Wannan Medical College (Wuhu, China) with a short history of a rapidly growing mass in the front of their neck, are reported. Both patients had undergone ultrasound examination, and the subsequent histopathological and immunohistochemical examinations confirmed that the two masses were primary diffuse large B-cell lymphoma. The ultrasonographic findings of these two cases are discussed in the present report.
\end{abstract}

\section{Introduction}

Primary thyroid lymphoma (PTL) is a relatively rare malignant tumor, which accounts for $<5 \%$ of all thyroid malignancies and $\sim 3 \%$ of all extranodal lymphomas (1). The two most common subtypes of PTL are diffuse large B-cell lymphoma (DLBCL) and mucosa-associated lymphoid tissue (1). Other subtypes of PTL include follicular lymphoma and classic Hodgkin's lymphoma of T-cell origin (2). Clinical signs of PTL include a rapidly enlarging mass in the neck, hoarseness and dyspnea; other symptoms, such as weight loss and fever, may also be present. The treatment and prognosis of PTL depend on the specific subtype and staging. A 5-year survival of $90 \%$ has been reported in correctly diagnosed and treated PTL patients (2), so a timely and accurate diagnosis of PTL is required for successful treatment and a good prognosis. Aggressive PTL is

Correspondence to: Dr Guobing Hu, Department of Ultrasound, The First Affiliated Hospital of Wannan Medical College, 2 West Zheshan Road, Wuhu, Anhui 241001, P.R. China

E-mail: guobing.hu@163.com

Key words: thyroid, diffuse large B-cell lymphoma, mass, ultrasonography rare, and there is limited literature regarding its imaging characteristics (3). In the present study, two cases of PTL in two elderly patients are reported. Both cases presented as enlarged thyroid and were associated with extensive invasion into adjacent structures. The ultrasonographic features of the two cases are described in the present report. Written informed consent was obtained from both patients.

\section{Case reports}

Case 1. In August 2014, 63-year-old female patient presented to The First Affiliated Hospital of Wannan Medical College (Wuhu, China) with a 2-month history of a rapidly growing mass in the front of her neck. The patient had no symptoms of dyspnea, hoarseness, dysphagia, dysphasia or stridor. On physical examination, the right thyroid lobe was noted to be enlarged and firm. There was a palpable mass in the front of her neck, which moved during swallowing. The patient had no history of Hashimoto's thyroiditis (HT), and her thyroid function test was within the normal range [free T3 (FT3), $4.89 \mathrm{pmol} / \mathrm{l}$; normal range, 3.5-6.5 pmol/1; free T4 (FT4), $18.73 \mathrm{pmol} / \mathrm{l}$; normal range, 11.5-22.7 pmol/1; ultrasensitive thyroid stimulating hormone (TSH3UL), $0.591 \mathrm{milli}$ international units (mIU)/1; normal range, 0.55-4.78 mIU/1; antithyroglobulin antibody (ATG-Ab), 25.90; normal range, 0-60 units (U)/ml; thyroid peroxidase antibody (TPO-Ab), $38.70 \mathrm{U} / \mathrm{ml}$; normal range, 0-60 U/ml]. Ultrasonography (US; Mylab 90; Esaote, Genoa, Italy) revealed an irregular shaped mass in the right lobe of the thyroid. The mass, which measured $\sim 6 \times 5 \mathrm{~cm}$ in size, extended to the right submandibular region and invaded the right internal jugular vein (RIJV) and adjacent muscles (Fig. 1A). The mass was heterogeneously hypoechoic. There were increased twisted blood flow signals, but no defined calcification, hemorrhage or necrotic portion were observed within the mass, which exhibited indistinct margins and posterior acoustic enhancement (PAE) (Fig. 1B). The subsequent surgery performed on the patient confirmed the presence of the mass in the right lobe of the thyroid. Lymphadenopathies invaded the RIJV, which was partly occluded, and the adjacent muscles. Therefore, the surgery included thyroidectomy, and resection of part of the RIJV and adjacent muscles. Postoperative histopathology and immunohistochemical examinations using a BX53 microscope (Olympus Corporation, Tokyo, Japan) confirmed primary DLBCL (Fig. 2): Hematoxylin and eosin (Wuxi Jiangyuan 

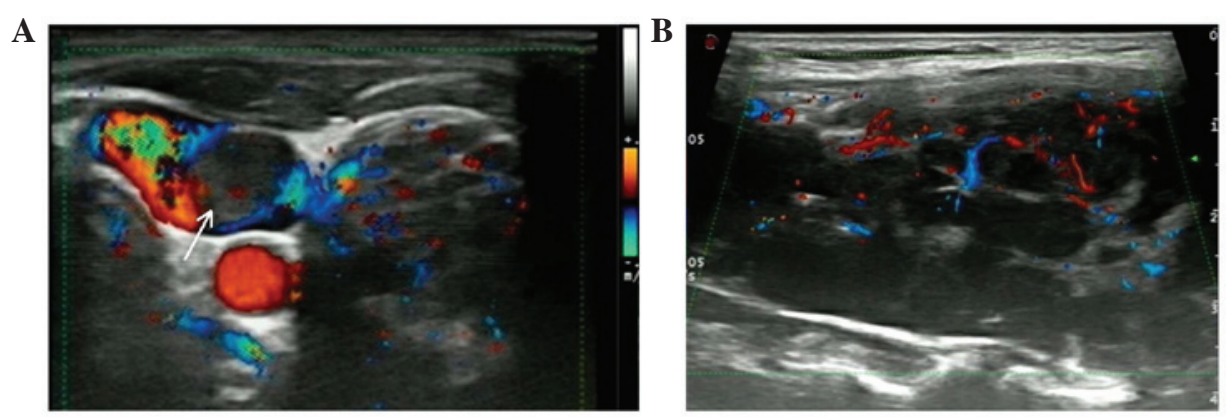

Figure 1. (A) Ultrasound examination revealed that the tumor thrombosis (white arrow) occluded part of the right internal jugular vein. (B) The mass was heterogeneously hypoechoic and occupied the entire right thyroid lobe. There were increased twisted blood flow signals, which are always detected in malignant tumors, but no defined calcification, hemorrhage or necrotic portion within the mass were observed, while posterior acoustic enhancement was clearly observed. Red and yellow signals indicate moderate and rapid blood flow towards the ultrasonic probe, respectively. Blue and green signals indicate moderate and rapid blood flow away from the ultrasonic probe, respectively.
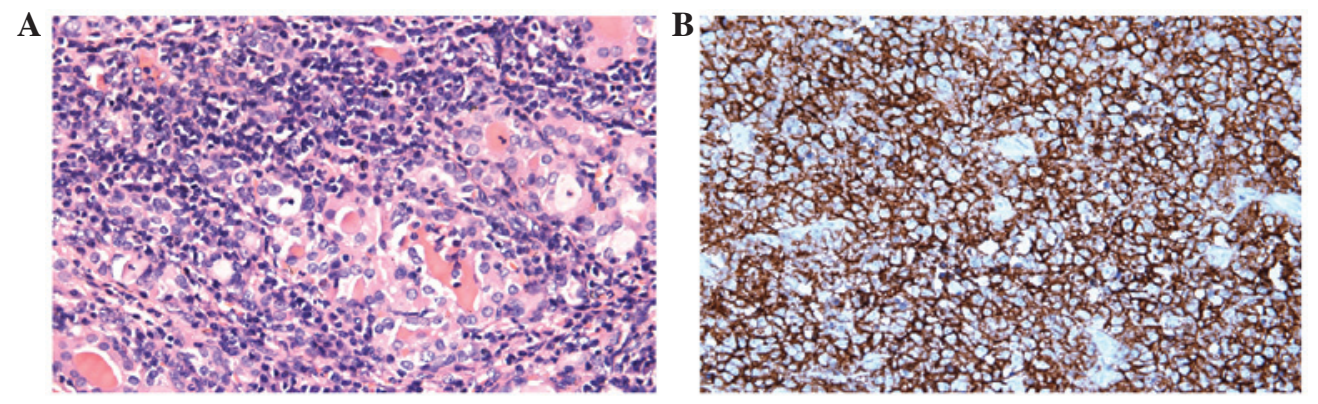

Figure 2. (A) Thyroid follicular atrophy was observed by hematoxylin and eosin staining, which was surrounded by a large number of diffuse large B-cell lymphoma cells. Magnification, $\mathrm{x} 400$. (B) Immunohistochemical staining demonstrated strong expression of cluster of differentiation 20 . Magnification, $\mathrm{x} 400$.
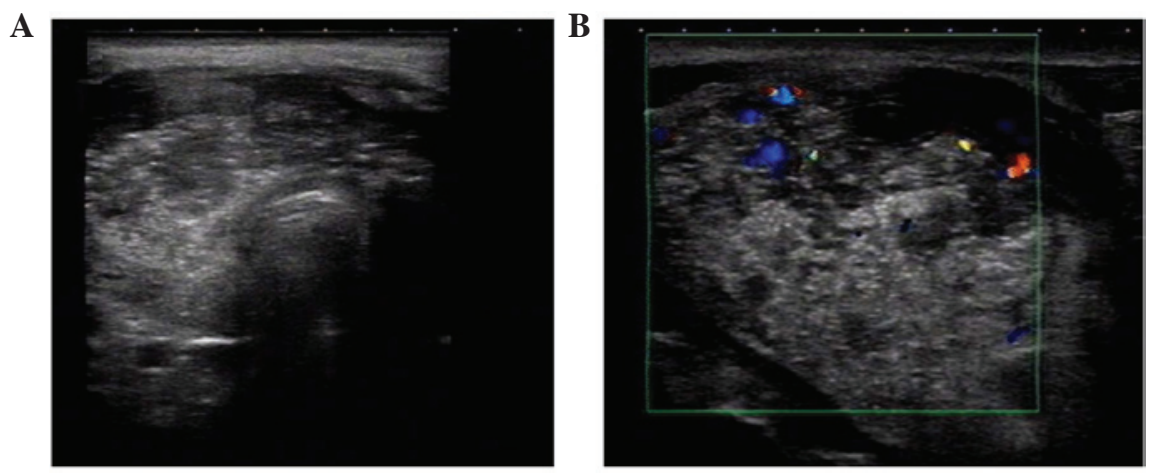

Figure 3. (A) Ultrasound (US) examination revealed that the thyroid was diffusely asymmetrically enlarged and exhibited unclear boundaries. The whole gland was non-uniformly hypoechoic. There were numerous calcified spots, but no defined hemorrhage or necrotic portion were present within the whole gland. (B) Color Doppler US revealed the presence of rare blood flow signals, which may be due to the small size of the tumor's blood vessels. Red and yellow signals indicate moderate and rapid blood flow towards the ultrasonic probe, respectively. Blue and green signals indicate moderate and rapid blood flow away from the ultrasonic probe, respectively.
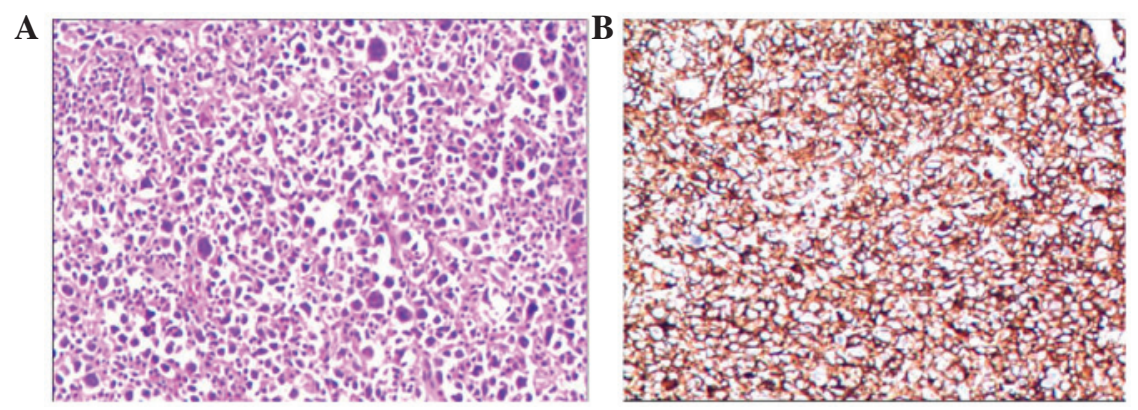

Figure 4. (A) Pathological confirmation of diffuse large B-cell lymphoma by hematoxylin and eosin staining. Magnification, x100. (B) Immunohistochemical analysis revealed that antigens associated with B cells, such as cluster of differentiation 20, were widely expressed. Magnification, x200. 
Industrial Technology and Trade Corporation, Wuxi, China) staining showed follicular atrophy of the thyroid and incubation of tissues with monoclonal mouse anti-human cluster of differentiation (CD)20 antibody (cat. no. 0001; 1:100; Fuzhou Maixin Biotechnology Development Co., Ltd., Fuzhou, China) at $25^{\circ} \mathrm{C}$ for 30 min revealed strong CD20 expression. The postoperative course was uneventful, and the patient was additionally treated with chemotherapy at the Second People's Hospital of Wuhu (Wuhu, China).

Case 2. In July 2013, a 60-year-old male patient was transferred to The First Affiliated Hospital of Wannan Medical College with a 1-month history of a rapidly enlarged thyroid and several nodules in the front of his neck. The patient had no symptoms of dyspnea, hoarseness, dysphagia, dysphasia or stridor. On physical examination, the whole thyroid was observed to be diffusely enlarged and firm. There were several palpable nodules in his neck, and the enlarged thyroid did not move during swallowing. The patient had no history of HT, and his thyroid function was markedly reduced (FT3, $1.52 \mathrm{pmol} / \mathrm{l}$; normal range, 3.5-6.5 pmol/1; FT4, $5.75 \mathrm{pmol} / \mathrm{l}$; normal range, 11.5-22.7 pmol/1; TSH3UL, $5.892 \mathrm{mIU} / 1$, normal range $0.55-4.78 \mathrm{mIU} / 1$; ATG-Ab, $37.20 \mathrm{U} / \mathrm{ml}$; normal range, 0-60 U/ml; TPO-Ab, $58.70 \mathrm{U} / \mathrm{ml}$; normal range, 0-60 U/ml). US revealed that the thyroid was diffusely asymmetrically enlarged with unclear boundaries, and the whole gland was non-uniformly hypoechoic. There were multiple calcified spots, but no defined hemorrhage or necrotic portion were observed within the whole gland (Fig. 3A). Color Doppler US displayed rare blood flow signals (Fig. 3B). A number of enlarged lymph nodes were detected, and the adjacent muscles in the front of his neck were invaded. Fine-needle aspiration biopsy (FNA; 22G, 70 mm needle; Hakko Medical Co., Ltd., Tokyo, Japan) and subsequent histopathology and immunohistochemical examinations using a BX53 microscope (Olympus Corporation) confirmed primary DLBCL (Fig. 4): Tissues were incubated with mouse anti-human CD20 antibody (cat. no. 0001; Fuzhou Maixin Biotechnology Development Co., Ltd.) at $25^{\circ} \mathrm{C}$ for $30 \mathrm{~min}$, which revealed that $\mathrm{CD} 20$ was widely expressed. The patient was only treated with chemotherapy at the Second People's Hospital of Wuhu (Wuhu, China).

\section{Discussion}

The most common clinical presentation of PTL is a rapidly growing goiter and other compressive symptoms, including dyspnea, hoarseness, dysphagia, dysphasia and stridor (4). The majority of patients with PTL are elderly women, and the condition is usually associated with HT (5). However, none of the present patients had compressive symptoms or HT. In addition, case 2 was a male patient. These characteristics rarely appear in the existing literature (6).

Computed tomography (CT) is generally used for evaluating the involvement of surrounding structures and displaying cervical and mediastinal nodal diseases in cases of PTL (7). Magnetic resonance imaging (MRI) is more accurate and sensitive than CT in diagnosing soft-tissue lesions (8), but positron emission tomography (PET) has been proved superior in diagnostic accuracy of PTL, compared with CT and MRI (9). However, the present cases had not undergone CT, MRI or PET, but only US, and the ultrasonographic findings are described in the present report.

US is the imaging modality of choice for thyroid pathology, and the typical ultrasonographic feature of PTL is the presence of a circumscribed mass or diffuse heterogeneous hypoechoic parenchyma with structures resembling septa (10). When presenting as a diffuse heterogeneous hypoechoic parenchyma, the ultrasonographic imaging features of PTL are very similar to those of HT (11). By contrast, cervical lymphadenopathies are normally present in the majority of patients with PTL, whereas calcification is never detected in HT and seldom detected in PTL, according to previous studies (12). The patient of case 2 had no history of HT, numerous enlarged lymph nodes were detected in his neck and multiple calcified spots were detected in his enlarged thyroid, which indicated malignancy. When presenting as a mass, PTL normally displays homogenous echogenicity, PAE, and lack of calcification, necrosis and cystic degeneration (13), while the surrounding structures are rarely invaded by PTL (12). Notably, PAE is the most common ultrasonographic feature of PTL, and it may also be detected in cystic lesions and solid benign tumors, but rarely detected in malignant masses (14). The ultrasonographic features of the patient of case 1 revealed a heterogeneous hypoechoic mass that invaded adjacent structures, including the RIJV and muscles. These features implied anaplastic thyroid carcinoma (ATC) rather than PTL. However, there was PAE and no defined calcification, hemorrhage or necrotic portion within the mass of the present case, which indicated PTL rather than ATC. Thus, it is difficult to distinguish PTL from ATC by US alone. Therefore, thyroid masses should undergo palpation-guided FNA biopsy, while non-palpable masses should undergo US-guided FNA biopsy for accurate diagnosis prior to surgical operation.

There is limited literature about blood flow signals in PTL. The blood flow signals in benign tumors are generally travelling naturally and present a uniform distribution, while malignant tumors are normally characterized by twisted blood flow signals and a non-uniform distribution (6). The characteristics of the blood flow signals in the patient of case 1 were coincident with those of malignant tumors. By contrast, color Doppler US of case 2 displayed rare blood flow signals, which may be due to the small size of the tumor's blood vessels, thus being difficult for color Doppler US to display blood flow signals, contrarily to what has been previously reported in the literature (13).

Due to its interference with gas and bone, US has difficulty in displaying organizational structure in the deep neck (14). However, the ultrasonographic imaging features of the present cases demonstrated clear invasion, which may be due to the fact that the invasion was superficial, and is indicative of malignant lesion in US. The tumor thrombosis of case 1 only occluded part of the RIJV lumen, thus the patient had no symptoms of RIJV occlusion. The tumor in case 2 only invaded the adjacent muscles, while the esophagus, trachea, carotid artery and jugular vein presented no sign of invasion.

In conclusion, the ultrasonographic features of PTL are complicated and variable. US is a valuable imaging method in assessing PTL, but it is difficult to establish a diagnosis by US alone. Therefore, pathology should be performed to confirm this disease. In summary, the present report describes the ultrasonographic features of two rare cases of PTL. 


\section{Acknowledgements}

The present study has been supported by funding from the First Affiliated Hospital of Wannan Medical College Program (grant no., WK2015ZF).

\section{References}

1. Walsh S, Lowery AJ, Evoy D, McDermott EW and Prichard RS Thyroid lymphoma: Recent advances in diagnosis and optimal management strategies. Oncologist 18: 994-1003, 2013.

2. Graff-Baker A, Roman SA, Thomas DC, Udelsman R and Sosa JA: Prognosis of primary thyroid lymphoma: Demographic, clinical, and pathologic predictors of survival in 1,408 cases. Surgery 146: 1105-1115, 2009.

3. Xia Y, Wang L, Jiang Y, Dai Q, Li X and Li W: Sonographic appearance of primary thyroid lymphoma-preliminary experience. PLoS One 9: e114080, 2014.

4. Sasai K, Yamabe H, Haga H, Tsutsui K, Dodo Y, Ishigaki T, Shibamoto Y and Abe M: Non-Hodgkin's lymphoma of the thyroid. A clinical study of twenty-two cases. Acta Oncol 35: 457-462, 1996.

5. Aozasa K: Hashimoto's thyroiditis as a risk factor of thyroid lymphoma. Acta Pathol Jpn 40: 459-468, 1990.

6. Wang Z, Fu B, Xiao Y, Liao J and Xie P: Primary thyroid lymphoma has different sonographic and color Doppler features compared to nodular goiter. J Ultrasound Med 34 317-323, 2015.

7. Li XB and Ye ZX: Primary thyroid lymphoma: Multi-slice computed tomography findings. Asian Pac J Cancer Prev 16: 1135-1138, 2015.
8. Takashima S, Nomura N, Noguchi Y, Matsuzuka F and Inoue T: Primary thyroid lymphoma: Evaluation with US, CT, and MRI. J Comput Assist Tomogr 19: 282-288, 1995.

9. Baba S, Abe K, Isoda T, Maruoka Y, Sasaki M and Honda H: Impact of FDG-PET/CT in the management of lymphoma. Ann Nucl Med 25: 701-716, 2011.

10. Kwak JY,Kim EK, Ko KH, Yang WI, Kim MJ, Son EJ, Oh KK and Kim KW: Primary thyroid lymphoma: role of ultrasound-guided needle biopsy. J Ultrasound Med 26: 1761-1765, 2007.

11. Nam M, Shin JH, Han BK, Ko EY, Ko ES, Hahn SY, Chung JH and Oh YL: Thyroid lymphoma: Correlation of radiologic and pathologic features. J Ultrasound Med 31: 589-594, 2012.

12. Vigliar E, Caleo A, Vitale M, Di Crescenzo V, Garzi A and Zeppa P: Early cytological diagnosis of extranodal stage I, primary thyroid Non-Hodgkin lymphoma in elderly patients. Report of two cases and review of the literature. BMC Surg 13 (Suppl 2): S49, 2013.

13. Ota H, Ito Y, Matsuzuka F, Kuma S, Fukata S, Morita S, Kobayashi K, Nakamura Y, Kakudo K, Amino N and Miyauchi A: Usefulness of ultrasonography for diagnosis of malignant lymphoma of the thyroid. Thyroid 16: 983-987, 2006.

14. Cho JH, Park YH, Kim WS, Oh SY, Cho SI, Kang HJ, $\mathrm{Na}$ II, Ryoo BY, Yang SH, Kim K, et al: High incidence of mucosa-associated lymphoid tissue in primary thyroid lymphoma: A clinicopathologic study of 18 cases in the Korean population. Leuk Lymphoma 47: 2128-2131, 2006. 